\title{
Procyanidin B2 inhibits high glucose-induced epithelial-mesenchymal transition in HK-2 human renal proximal tubular epithelial cells
}

\author{
DANDAN LI ${ }^{1}$, TINGBAO ZHAO ${ }^{1}$, JIANZHONG MENG ${ }^{2}$, YING JING ${ }^{2}$, FENGYU JIA $^{2}$ and PING HE ${ }^{2}$ \\ Departments of ${ }^{1}$ Spinal Cord Repair and ${ }^{2}$ Blood Purification, General Hospital of Jinan Military Region, \\ PLA, Jinan, Shandong 250031, P.R. China
}

Received November 4, 2014; Accepted July 30, 2015

DOI: $10.3892 / \mathrm{mmr} .2015 .4445$

\begin{abstract}
Diabetic nephropathy (DN) is not only an important chronic complication of diabetes, but is also one of the predominant cause of renal failure. Previous studies have indicated that the process termed 'epithelial-mesenchymal transition' (EMT) results in fibrosis of renal tubular epithelial cells, and is key in DN. As an antioxidant, procyanidin B2 can inhibit cardiac fibrosis; however, whether it has an effect on the inhibition of renal fibrosis remains to be elucidated. The present study demonstrated that high glucose levels were able to activate EMT-associated changes, including the loss of E-cadherin and increase in $\alpha$-smooth muscle actin ( $\alpha$-SMA), as determined by western blotting and immunofluorescence. Pre-treatment with procyanidin B2 reversed the high glucose-induced morphological changes, upregulated the expression of E-cadherin and downregulated the expression levels of vimentin and $\alpha$-SMA. Furthermore, procyanidin B2 decreased the phosphorylation of small mothers against decapentaplegic (Smad)2, Smad3 and P38, and upregulated the expression of phosphorylated-Smad7. In conclusion, the results of the present study suggested that
\end{abstract}

Correspondence to: Professor Tingbao Zhao, Department of Spinal Cord Repair, General Hospital of Jinan Military Region, PLA, 8 Lashan Road, Huaiyin, Jinan, Shandong 250031, P.R. China E-mail: tbaozhao_2014@163.com

Professor Jianzhong Meng, Department of Blood Purification, General Hospital of Jinan Military Region, PLA, 25 Shifan Road, Jinan, Shandong 250031, P.R. China

E-mail: meng2014_11@163.com

Abbreviations: HG, high glucose; DMEM, Dulbecco's modified Eagle's medium; FITC, fluorescein isothiocyanate; RIPA, radioimmunoprecipitation assay; TGF, transforming growth factor; EMT, epithelial-mesenchymal transition; SMA, smooth muscle cell actin, ROS, reactive oxygen species; MAPK, mitogen-activated protein kinase; DN, diabetic nephropathy; PBS, phosphate-buffered saline

Key words: procyanidin B2, epithelial-mesenchymal transition, HK-2, transforming growth factor- $\beta /$ small mothers against decapentaplegic, mitogen-activated protein kinase/P38 procyanidin B2 inhibited high glucose-induced EMT through the inhibition of transforming growth factor- $\beta /$ Smad and mitogen-activated protein kinase/P38 signaling pathways.

\section{Introduction}

Diabetic nephropathy (DN) is a chronic microvascular complication, which affects patients with diabetes. One of the most common characteristics of $\mathrm{DN}$ is diabetic glomerulosclerosis (1). A previous study demonstrated that the epithelial-mesenchymal transition (EMT)-like changes observed in podocytes are associated with podocyte detachment, which may result in focal glomerulosclerosis (2). Several studies have suggested that EMT, a process by which differentiated epithelial cells undergo a phenotypic conversion that gives rise to matrix-producing fibroblasts and myofibroblasts, may be important in the pathogenesis of diabetic kidney disease $(3,4)$. The high glucose $(\mathrm{HG})$-induced EMT of renal tubular epithelial cells is a key process in glomerulosclerosis and, mediated by factors, including E-cadherin and $\alpha$-smooth muscle actin ( $\alpha$-SMA), epithelial cells may lose their epithelial characteristics and gain mesenchymal cell properties $(5,6)$. However, the process of HG-induced EMT remains to be fully elucidated.

Procyanidins are the most abundant polyphenols present in red wine, and they are also present in cereals, fruits, chocolate and tea (7). Several epidemiological studies have demonstrated that procyanidin B2 can inhibit hepatic and cardiac fibrosis $(8,9)$. Based on the possible anti-fibrotic activity of procyanidins, the present study investigated whether procyanidins inhibit HG-induced EMT in the early stage of diabetic kidney glomerulosclerosis.

The transforming growth factor (TGF)- $\beta /$ small mothers against decapentaplegic (Smad) and mitogen-activated protein kinase (MAPK)/P38 signaling pathways are involved in promoting EMT and are associated with the development of DN $(10,11)$. The aim of the present study was to determine the role of the MAPK/P38 and TGF- $\beta /$ Smad signaling pathways in HG-induced EMT, and to examine the changes in the two pathways in HK-2 cells cultured with procyanidin B2. The detailed investigation of this plant extract may provide a novel therapeutic strategy for the treatment of DN. 


\section{Materials and methods}

Reagents. Dulbecco's modified Eagle's medium (DMEM), penicillin-streptomycin $(5,000 \mathrm{U} / \mathrm{ml}$ penicillin; $5,000 \mathrm{U} / \mathrm{ml}$ streptomycin) and fetal bovine serum (FBS) were purchased from Gibco Life Technologies (Grand Island, NY, USA). D-glucose (analytical grade) was purchased from Guanghua Chemical Factory Co., Ltd. (Guangdong, China). Procyanidin B2 and Triton X-100 were purchased from Sigma-Aldrich (St. Louis, MO, USA). The anti- $\beta$-actin, anti-P38, anti-phosphorylated (p)-P38 and anti-p-Smad 2, 3 and 7 antibodies were purchased from Santa Cruz Biotechnology, Inc. (Dallas, TX, USA). The anti-fibronectin (FN), anti- $\alpha$-SMA, anti-E-cadherin and anti-vimentin antibodies were purchased from BD Biosciences (Franklin Lakes, NJ, USA). An enhanced chemiluminescence (ECL) kit was purchased from Pierce Biotechnology, Inc. (Rockford, IL, USA). All reagents used were trace element analytical grade and all water used was glass distilled.

Cell culture. The HK-2 human renal proximal tubular epithelial cell line (CRL-2190; American Type Culture Collection, Danvers, MA, USA) was cultured at a density of $1 \times 10^{5}$ cells $/ \mathrm{ml}$ in DMEM supplemented with $5.5 \mathrm{mmol} / \mathrm{l} \mathrm{D}$-glucose (normal glucose; NG) and $10 \% \mathrm{FBS}$ at $37^{\circ} \mathrm{C}$ in a humidified atmosphere containing $5 \% \mathrm{CO}_{2}$. The cells were subcultured every 3 days using $0.2 \%$ trypsin (Sigma-Aldrich) with $0.02 \%$ EDTA (Sigma-Aldrich). The near confluent HK-2 cells (80\%) were subsequently transferred into serum-free DMEM for overnight starvation prior to each experiment. In order to induce EMT, the cells were cultured in high glucose $(\mathrm{HG})$ medium containing $60 \mathrm{mmol} / \mathrm{l} \mathrm{D}$-glucose for $48 \mathrm{~h}$ at $37^{\circ} \mathrm{C}$. The concentration was selected based on previous studies $(12,13)$. To study the protective effects of procyanidin $\mathrm{B} 2, \mathrm{HK}-2$ cells were incubated with $10 \mu \mathrm{M}$ procyanidin $\mathrm{B} 2$ at $37^{\circ} \mathrm{C}$ in a humidified atmosphere for $48 \mathrm{~h}$.

ELISA. The protein expression levels of TGF- $\beta$ were measured using a TGF- $\beta$ ELISA kit (R\&D Systems, Minneapolis, MN, USA). Briefly, the HK-2 cells were seeded at a density of $4 \times 10^{5}$ cells/well into a 6 -well plate and cultured for $24 \mathrm{~h}$ at $37^{\circ} \mathrm{C}$. The supernatants were collected from the HK- 2 cell cultures for ELISA. The secreted protein concentration of TGF- $\beta$ per $10^{5}$ cells was measured and calculated from the standard curve using the ELISA kit. Briefly, $100 \mu \mathrm{l}$ samples were added to sample diluent and incubated for $1 \mathrm{~h}$ at $37^{\circ} \mathrm{C}$ with agitation, following washing with washing buffer (Sigma-Aldrich). Enzyme-conjugated secondary antibody was added to the wells and incubated for $2 \mathrm{~h}$ at $37^{\circ} \mathrm{C}$ prior to addition of the substrate solution. Absorbance was measured using an ELISA reader (Multiskan MK3; Thermo Fisher Scientific, Inc., Waltham, MA, USA) at a wavelength of $450 \mathrm{~nm}$.

Western blot analysis. The cells were collected and washed with phosphate-buffered saline (PBS) prior to being lysed on ice for $30 \mathrm{~min}$ in lysis buffer (Beyotime Institute of Biotechnology, Haimen, China) containing $50 \mathrm{mM}$ Tris (pH 8.1), $1 \%$ SDS, sodium pyrophosphate, $\beta$-glycerophosphate, sodium orthovanadate, sodium fluoride, EDTA, and leupeptin, and centrifuged at $15,000 \mathrm{xg}$ for $30 \mathrm{~min}$ at room temperature.
The supernatants were collected, and the protein concentration was determined using a Bicinchoninic Acid Protein Assay kit (Pierce Biotechnology, Inc.). The protein were boiled for $5 \mathrm{~min}$ and $10 \mu \mathrm{g}$ total protein was loaded into the appropriate well to be separated by $10 \%$ SDS-PAGE (Beyotime Institute of Biotechnology). The proteins on the gel were then transferred onto a nitrocellulose membranes (EMD Millipore, Billerica, MA, USA) using a Bio-Rad A101441 apparatus (Bio-Rad Laboratories, Inc., Hercules, CA, USA) for $2 \mathrm{~h}$ at $4^{\circ} \mathrm{C}$ and $100 \mathrm{~V}$. The protein-bound membranes were then blocked and washed in Tris-buffered saline with $20 \%$ Tween 20 (Sigma-Aldrich). The nitrocellulose membranes were cut according to the molecular weight of the protein, and were incubated with antibodies. The following primary antibodies were used: Anti- $\beta$-actin mouse monoclonal IgG1 (1:400; cat. no. sc-8432; Santa Cruz Biotechnology, Inc.; 24 h incubation at $\left.4^{\circ} \mathrm{C}\right)$, anti- $\alpha$-SMA goat polyclonal $\operatorname{IgG}(1: 400$; cat. no. sc-324317; Santa Cruz Biotechnology, Inc.; 24 h incubation at $4^{\circ} \mathrm{C}$ ), anti-E-cadherin mouse monoclonal IgG1 (1:400; cat. no. sc-52327; Santa Cruz Biotechnology, Inc.; $24 \mathrm{~h}$ incubation at $4^{\circ} \mathrm{C}$ ), anti-FN mouse monoclonal IgG1 (1:400; cat. no. sc-52331; Santa Cruz Biotechnology, Inc.; 24 h incubation at $4^{\circ} \mathrm{C}$ ), anti-vimentin mouse monoclonal IgG1 (1:400; cat. no. sc-373717; Santa Cruz Biotechnology, Inc.; $24 \mathrm{~h}$ incubation at $4^{\circ} \mathrm{C}$ ), anti-P38 mouse monoclonal IgG1 (1:400; cat. no. sc-33688; Santa Cruz Biotechnology, Inc.; 24 h incubation at $\left.4^{\circ} \mathrm{C}\right)$, anti-p-P38 mouse monoclonal IgG1 (1:400; cat. no. sc-7973; Santa Cruz Biotechnology, Inc.; 24 h incubation at $\left.4^{\circ} \mathrm{C}\right)$, anti-p-Smad 2 mouse monoclonal IgG1 (1:400; cat. no. sc-393312; Santa Cruz Biotechnology, Inc.; 24 h incubation at $4^{\circ} \mathrm{C}$ ), anti-p-Smad 3 mouse monoclonal IgG1 (1:400; cat. no. sc-101154; Santa Cruz Biotechnology, Inc.; $24 \mathrm{~h}$ incubation at $4^{\circ} \mathrm{C}$ ) and anti-p-Smad 7 mouse monoclonal IgG1 (1:400; cat. no. sc-365846; Santa Cruz Biotechnology, Inc.; $24 \mathrm{~h}$ incubation at $4^{\circ} \mathrm{C}$ ). The membranes were then incubated with goat anti-mouse IgG2a-B peroxidase-conjugated secondary antibodies (1:400; cat. no. sc-2073; Santa Cruz Biotechnology, Inc.; $24 \mathrm{~h}$ incubation at $4^{\circ} \mathrm{C}$ ). The blots were visualized using an ECL kit (Pierce Biotechnology, Inc.) and the relative quantities of the proteins were analyzed. The results were quantified using Quantity One software V4.62 (Bio-Rad Laboratories, Inc.).

Fluorescence microscopy. The cells $\left(1 \times 10^{5}\right.$ cells $\left./ \mathrm{ml}\right)$ were washed once with ice-cold PBS and fixed with $4 \%$ paraformaldehyde (Sigma-Aldrich) for $30 \mathrm{~min}$ at $4^{\circ} \mathrm{C}$. Following being washed three times with PBS, the cells were incubated with $1 \%$ Triton X-100 for $10 \mathrm{~min}$. The cells were blocked at nonspecific antibody binding sites by incubating in PBS containing $0.3 \%$ Triton X-100 and $0.5 \%$ bovine serum albumin (Sigma-Aldrich) for $30 \mathrm{~min}$ at room temperature. The cells were subsequently incubated with an antibody targeting E-cadherin or $\alpha$-SMA (1:200) overnight at room temperature. Subsequently, the cells were incubated with a fluorescein isothiocyanate- or tetramethylrhodamine-conjugated immunoglobulin $\mathrm{G}$ antibody (1:100 in PBS) for $0.5 \mathrm{~h}$ at room temperature. Hoechst 33342 (10 $\mu \mathrm{g} / \mathrm{ml}$; Sigma-Aldrich) was then added to the cells for $15 \mathrm{~min}$ at room temperature. Following three washes with PBS, the cells were visualized under a fluorescence microscope (Olympus SZ51; Olympus Corporation, Tokyo, Japan). 
A

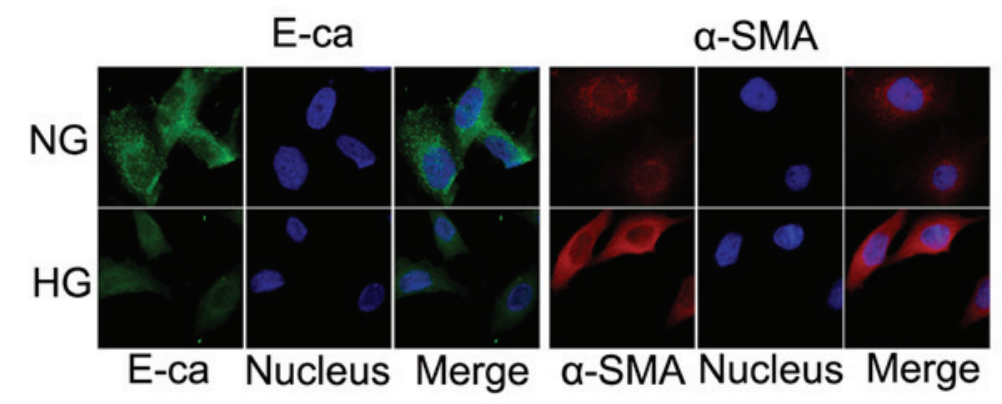

B
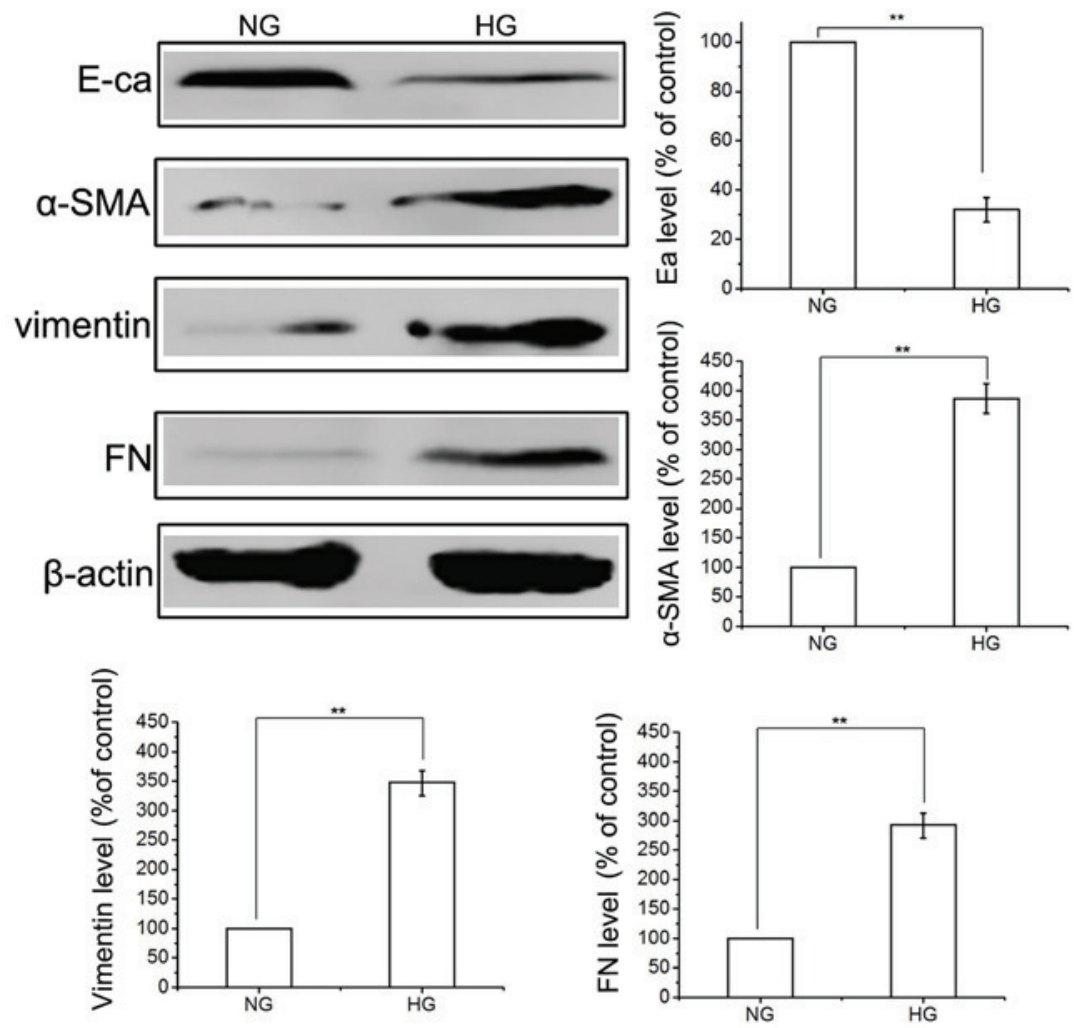

Figure 1. HG promotes epithelial-to-mesenchymal transition in HK-2 cells. (A) HK-2 cells were incubated in NG or HG conditions for 48 h, and the expression levels of E-ca and ( $\alpha$-SMA) were detected using immunofluorescence. (B) Cells were treated, as described, and the expression levels of E-ca, $\alpha$-SMA, FN and vimentin were detected using western blotting. The results are representative of three independent experiments. $\beta$-actin was used as a loading control. Data are expressed as the mean \pm standard error of the mean. ${ }^{* *} \mathrm{P}<0.01$, vs. NG group. HG, high glucose; NG, normal glucose; E-ca, E-cadherin; $\alpha$-SMA, $\alpha$-smooth muscle actin; FN, fibronectin.

Statistical analysis. Data are expressed as the mean \pm standard error of the mean. Variance was homogenous enabling use of the standard one-way analysis of variance (ANOVA) methodology. On establishment of statistical significance using ANOVA, individual comparisons were made using Tukey's multiple comparison test. $\mathrm{P}<0.05$ was considered to indicate a statistically significant difference. Statistical analyses were performed using SPSS version 18 (SPSS, Inc., Chicago, IL, USA).

\section{Results}

$H G$ induces HK-2 cells to undergo EMT. To determine whether HG induced EMT, the HK-2 cells were incubated in NG (5.5 mmol/l D-glucose) or HG (60 mmol/l D-glucose) conditions. Initially, exposure of the HK-2 cells to HG for $48 \mathrm{~h}$ decreased the protein expression levels of E-cadherin and increased the expression levels of $\alpha$-SMA, compared with the NG group, determined by immunofluorescence (Fig. 1A). In addition, HG-induced EMT was confirmed by western blotting, which indicated the upregulation of $\alpha$-SMA, FN and vimentin and the downregulation of E-cadherin (Fig. 1B). These results suggested that an HG environment activated the EMT process in the HK-2 cells.

Effects of procyanidin B2 on the expression of EMT-associated proteins in $H G$-induced $H K-2$ cells. To determine the effects of procyanidin $\mathrm{B} 2$ in EMT, the HK-2 cells were treated with or without $60 \mathrm{mmol} / \mathrm{l} \mathrm{D}$-glucose for $48 \mathrm{~h}$, in the presence or absence of procyanidin B2. The expression levels of FN, 
$\mathbf{A}$
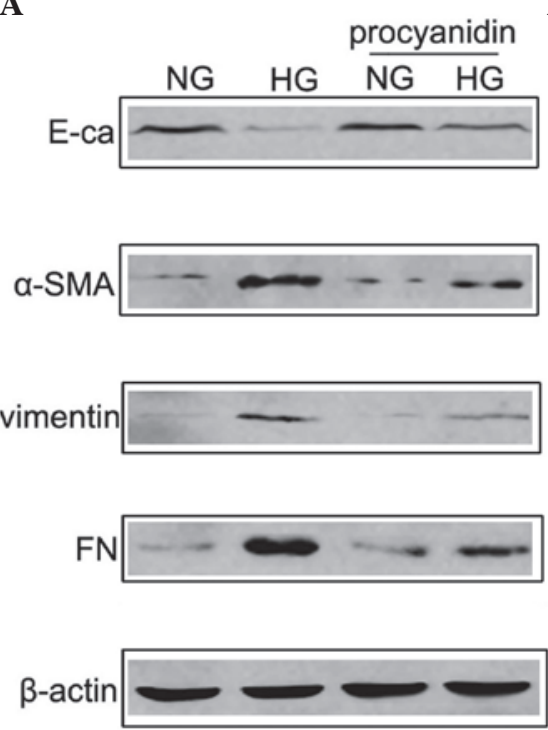

B
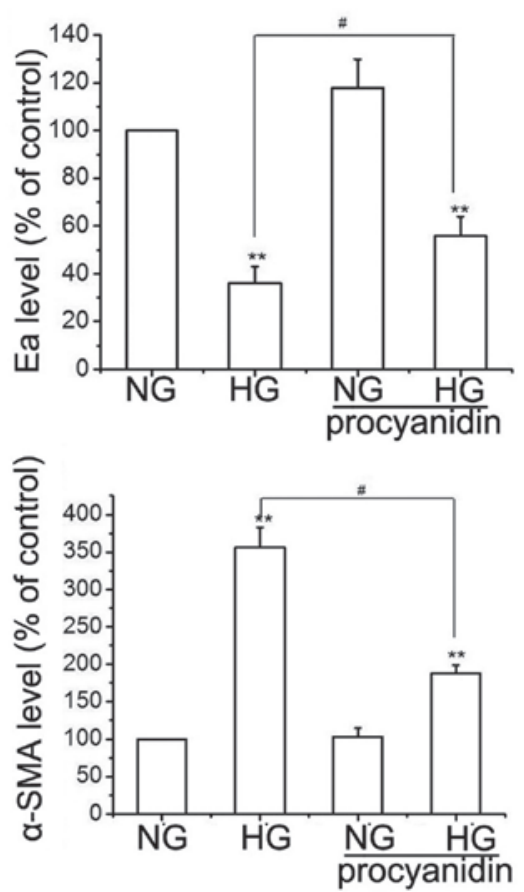
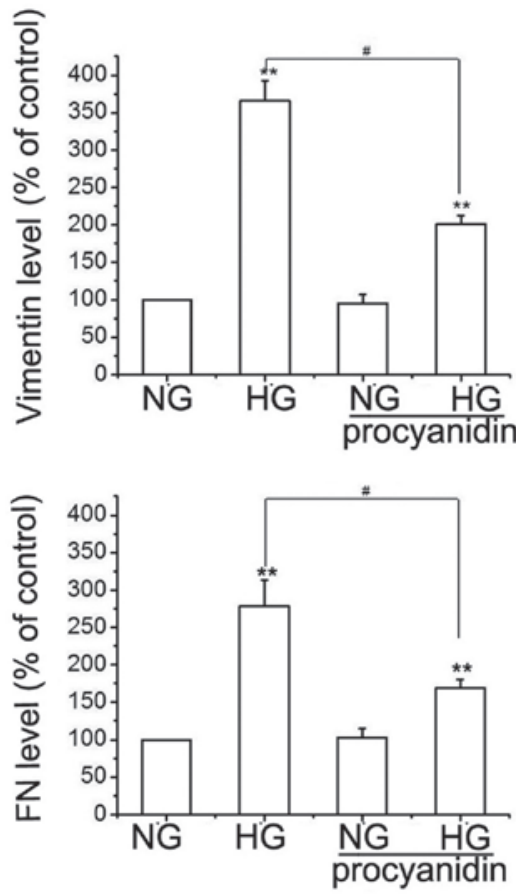

Figure 2. Procyanidin B2 inhibits HG-induced epithelial-to-mesenchymal transition in HK-2 cells. (A) HK-2 cells were incubated in NG or HG conditions in the presence or absence of procyanidin $\mathrm{B} 2$ for $48 \mathrm{~h}$, and the expression levels of E-ca, $\alpha$-SMA, FN and vimentin were detected using western blotting. (B) Results are representative of three independent experiments. $\beta$-actin was used as a loading control. Data are expressed as the mean \pm standard error of the mean $\left({ }^{* *} \mathrm{P}<0.01\right.$, vs. NG group; ${ }^{\text {P }}<0.01$, HG vs. HG+procyanidin B2 group). HG, high glucose; NG, normal glucose; E-ca, E-cadherin; $\alpha$-SMA, $\alpha$-smooth muscle actin; FN, ibronectin.

A

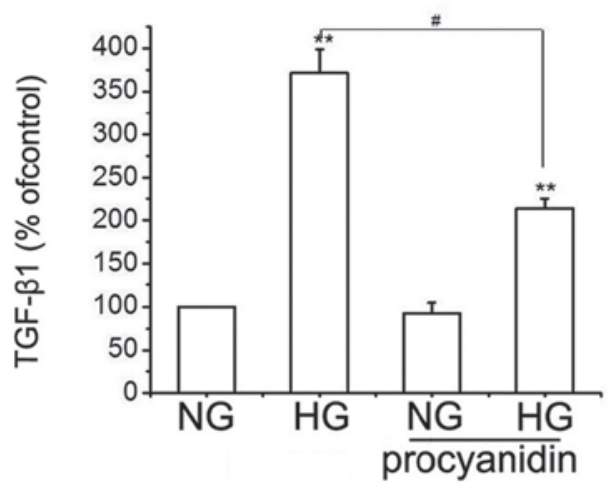

C

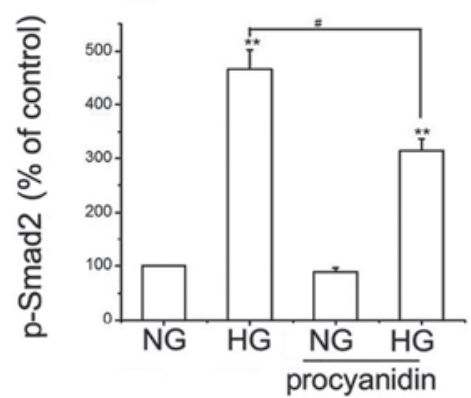

D
B
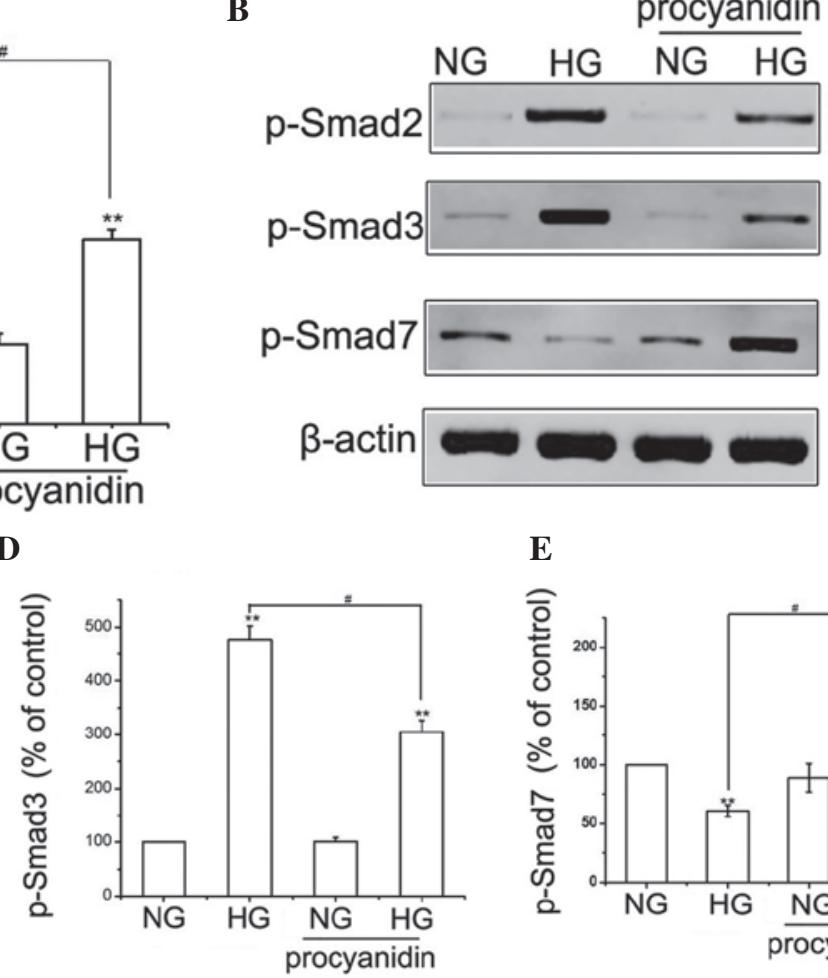

$\mathbf{E}$

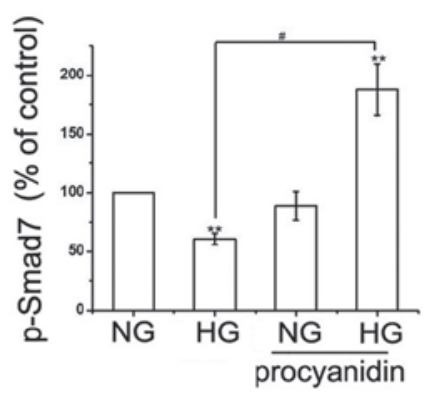

Figure 3. Procyanidin B2 inhibits HG-induced epithelial-to-mesenchymal transition via the TGF- $\beta$ /Smads signaling pathway in HK-2 cells. (A) HK-2 cells were incubated in NG or HG conditions in the presence or absence of procyanidin B2 for $48 \mathrm{~h}$, and the protein expression levels of TGF- $\beta$ were measured using ELISA. The results are representative of three independent experiments. ${ }^{* *} \mathrm{P}<0.01$, vs. NG group; ${ }^{\text {}} \mathrm{P}<0.01, \mathrm{HG}$ vs. HG+procyanidin $\mathrm{B} 2$ group. (B) Cells were treated, as described, and the expression levels of p-Smad2, 3 and 7 were detected using western blotting. (C-E) Results are representative of three independent experiments. $\beta$-actin was used as a loading control. ${ }^{* *} \mathrm{P}<0.01$, vs. NG group; ${ }^{\# \mathrm{P}}<0.01, \mathrm{HG}$ vs. HG+procyanidin $\mathrm{B} 2$ group. Data are expressed as the mean \pm standard error of the mean. HG, high glucose; NG, normal glucose; TGF, transforming growth factor; Smad, small mothers against decapentaplegic; p-, phosphorylated. 
$\mathbf{A}$
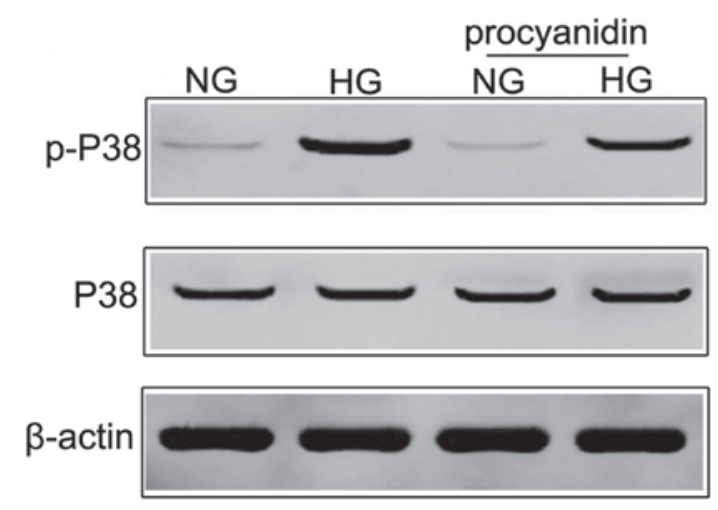

B

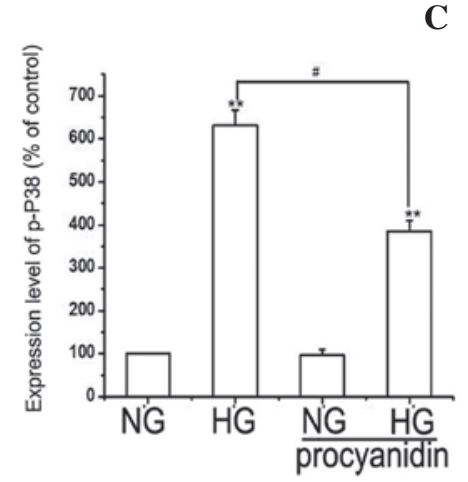

C

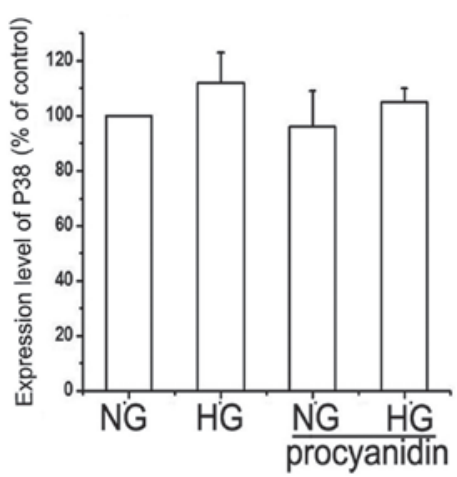

Figure 4. Procyanidin B2 inhibits HG-induced epithelial-to-mesenchymal transition via the mitogen-activated protein kinase/P38 signaling pathway in HK-2 cells. (A) HK-2 cells were incubated in NG or HG conditions in the presence or absence of procyanidin B2 for 48 h, and the expression levels of P38 and p-P38 were detected using western blotting. (B and C) Results are representative of three independent experiments. $\beta$-actin was used as a loading control. Data are expressed as the mean \pm standard error of the mean. ${ }^{* *} \mathrm{P}<0.01$, vs. NG group; ${ }^{*} \mathrm{P}<0.01$, HG vs. HG+procyanidin $\mathrm{B} 2$ group. HG, high glucose; NG, normal glucose; p-, phosphorylated.

E-cadherin, vimentin and $\alpha$-SMA were detected using western blotting. As shown in Fig. 2, exposure of the HK-2 cells to HG for $48 \mathrm{~h}$ decreased the protein expression levels of E-cadherin and increased the expression levels of FN, $\alpha$-SMA and vimentin, compared with the NG group. However, HG-induced EMT was attenuated when the HK-2 cells were pre-treated with $10 \mu \mathrm{M}$ procyanidin $\mathrm{B} 2$, which was indicated by the reduced expression levels of FN, $\alpha$-SMA and vimentin, and the increased expression of E-cadherin (Fig. 2). The concentration of procyanidin B2 was selected based on a previous study (14). These results suggested that procyanidin B2 supplementation reversed HG-induced EMT in the HK-2 cells.

Effects of procyanidin B2 on the expression of TGF- $\beta /$ Smad in $H K-2$ cells. The TGF- $\beta /$ Smads signaling pathway is considered to contribute to the development of DN by increasing glomerulosclerosis and inducing EMT $(15,16)$. To determine the effects of procyanidin B2 on the expression of TGF- $\beta$ in HG-treated HK-2 cells, the protein expression of TGF- $\beta$ was measured using ELISA (Fig. 3A). Compared with the NG group, the expression of TGF- $\beta$ was significantly higher in the HG-treated group; however, treatment with procyanidin $\mathrm{B} 2$ reduced the HG-induced expression of TGF- $\beta$. Furthermore, the effects of procyanidin B2 on HG-induced expression of Smads in the HK-2 cells were examined using western blotting. HG treatment resulted in a substantial increase in the expression levels of $\mathrm{p}-\mathrm{Smad} 2$ and $\mathrm{p}-\mathrm{Smad} 3$, and decreased the protein expression of p-Smad7 in the HK-2 cells. However, procyanidin B2 pre-treatment significantly attenuated HG-induced expression of $\mathrm{p}-\mathrm{Smad} 2$ and $\mathrm{p}-\mathrm{Smad} 3$, and increased the expression of p-Smad7 in the HK-2 cells (Fig. 3B-E). These results indicated that procyanidin B2 inhibited HG-induced EMT via the TGF- $\beta$ /Smads signaling pathway.

Effects of procyanidin $B 2$ on the expression of MAPK/P38 in $H K-2$ cells. The MAPK/P38 signaling pathway has previously been reported to be involved in EMT (17); however, whether procyanidin B2 exerts its effect on EMT in HK-2 cells via this pathway remains to be elucidated. Therefore, the present study examined the effect of procyanidin B2 supplementation on the MAPK/P38 pathway. Compared with the NG group, the expression of p-P38 was upregulated by $\mathrm{HG}$, whereas the expression levels of total P38 were not significantly altered. Notably, pre-treatment of the HK-2 cells with procyanidin B2 significantly inhibited the HG-induced expression of p-P38. However, no significant difference was observed in the expression of total P38 in the HG+procyanidin B2 group, compared with the HG group (Fig. 4). These results suggested that procyanidin B2 may be involved in HG-induced EMT via regulation of the MAPK/P38 pathway.

\section{Discussion}

The pathogenesis of DN is the result of several factors, and insulin metabolism disorder as a result of long-term high blood sugar is considered the predominant cause of DN (18). 
Renal hemodynamic changes caused by high blood sugar, and the series of consequences of abnormal glucose metabolism are the basis of kidney disease (19). HG-induced EMT is an important process, leading to glomerulosclerosis and tubulointerstitial fibrosis (5). EMT is described as the loss of epithelial characteristics from epithelial cells, and the acquisition of properties typical of mesenchymal cells. EMT facilitates cell movement and the generation of novel tissue types during development, and also contributes to the pathogenesis of disease $(20,21)$. The characteristics of EMT essential to wound healing also link EMT to organ fibrosis, including pulmonary, renal and hepatic fibrosis (20). A previous study demonstrated that tubular epithelium can transdifferentiate into fibroblasts via the process of EMT, which is regarded as an important event in the pathogenesis of tubulointerstitial fibrosis (22). The findings of the present study were consistent with these previous results. HG-induced EMT was confirmed using western blotting, which detected the upregulation of $\alpha$-SMA, FN and vimentin, and the downregulation of E-cadherin, associated with a transition from the epithelial phenotype of HK-2 cells to a myofibroblastic phenotype.

Proanthocyanidins are a class of substances, which are linked by catechin, epicatechin and gallate (23). Procyanidin B2 is a biologically active component of grape seeds, which has been reported to possess various pharmacological and biochemical effects (24). The effects of procyanidin B2 on various types of cancer have been reported $(25,26)$; however, the effects of procyanidin B2 on EMT in human renal proximal tubular epithelial cells remain to be fully elucidated. The present study investigated the effects of procyanidin B2 on HG-induced EMT, and examined the underlying mechanisms. The occurrence of oxidative stress is key in EMT (27), and procyanidin B2, which is present in grape seeds, apples and cacao beans, has antioxidant properties (28). Therefore, the present study hypothesized that procyanidin B2 may have an inhibitory effect on EMT. The results of the present study demonstrated that procyanidin B2 treatment provided effective protection against $\mathrm{HG}$-induced EMT, evidenced by a decrease in the upregulation of vimentin and $\alpha$-SMA, and the amelioration of E-cadherin in the human renal proximal tubular epithelial cell line (Fig. 2).

TGF- $\beta$ has been described as a 'master switch' regulating EMT, and has been demonstrated to signal primarily via the Smad $2 / 3$ pathway (29). Smad7 is crucial in antagonizing EMT induced by TGF- $\beta$ signaling (30). The present study demonstrated that procyanidin B2 pre-treatment significantly attenuated HG-induced EMT by directly downregulating p-Smad 2 and 3, and indirectly upregulating p-smad7; accompanied by a decrease in the upregulation of $\mathrm{FN}, \alpha-\mathrm{SMA}$ and vimentin, and an increase in the downregulation of E-cadherin. The downstream effects of TGF- $\beta$ may not only be as a consequence of the Smads signaling pathway, but may be the result of multiple signaling pathways, acting to modulate the effects of TGF- $\beta$ signaling. Activation of the MAPK/P38 signaling pathway is not exclusive to TGF- $\beta$ and may be activated to induce EMT (31). The present study also demonstrated that procyanidin B2 significantly inhibited the HG-induced expression of p-P38 and weakened HG-induced EMT.

In conclusion, the results of the present study demonstrated that procyanidin B2 inhibited HG-induced EMT, most likely via inhibition of the expression of TGF- $\beta, \mathrm{p}-\mathrm{Smad} 2$ and 3 , as well as the P38/MAPK signaling pathway, in HK-2 cells. The identification of procyanidin B2 as an inhibitor of HG-induced EMT represents an important finding. Further investigations may examine this target for limiting EMT and for the treatment of DN.

\section{Acknowledgements}

The present study was supported by the Military Logistics Research Programs of China (grant no. CJN10L075) and by the China Postdoctoral Science Foundation (grant no. 2012M512175).

\section{References}

1. Zhao HL, Lai FM, Tong PC, Tomlinson B and Chan JC: Clinicopathologic characteristics of nodular glomerulosclerosis in Chinese patients with type 2 diabetes. Am J Kidney Dis 44: 1039-1049, 2004.

2. Samejima K, Nakatani K, Suzuki D, Asai O, Sakan H, Yoshimoto S, Yamaguchi Y, Matsui M, Akai Y, Toyoda M, et al: Clinical significance of fibroblast-specific protein-1 expression on podocytes in patients with focal segmental glomerulosclerosis. Nephron Clin Pract 120: c1-c7, 2012.

3. Liu Y: New insights into epithelial-mesenchymal transition in kidney fibrosis. J Am Soc Nephrol 21: 212-222, 2010.

4. Carew RM, Wang B and Kantharidis P: The role of EMT in renal fibrosis. Cell Tissue Res 347: 103-116, 2012.

5. Li J, Kang SW, Kim JL, Sung HY, Kwun IS and Kang YH: Isoliquiritigenin entails blockade of TGF-beta1-SMAD signaling for retarding high glucose-induced mesangial matrix accumulation. J Agric Food Chem 58: 3205-3212, 2010.

6. Tang L, Li H, Gou R, Cheng G, Guo Y, Fang Y and Chen F: Endothelin-1 mediated high glucose-induced epithelial-mesenchymal transition in renal tubular cells. Diabetes Res Clin Pract 104: 176-182, 2014.

7. Quesada IM, Del Bas JM, Bladé C, Ardèvol A, Blay M, Salvadó MJ, Pujadas G, Fernández-Larrea J and Arola L: Grape seed procyanidins inhibit the expression of metallothione in genes in human HepG2 cells. Genes Nutr 2: 105-109, 2007.

8. Palfi A, Bartha E, Copf L, Mark L, Gallyas F Jr, Veres B, Kalman E, Pajor L, Toth K, Ohmacht R and Sumegi B: Alcohol-free red wine inhibits isoproterenol-induced cardiac remodeling in rats by the regulation of Akt1 and protein kinase C alpha/beta II. J Nutr Biochem 20: 418-425, 2009.

9. Choi JH, Hwang YP, Choi CY, Chung YC and Jeong HG: Anti-fibrotic effects of the anthocyanins isolated from the purple-fleshed sweet potato on hepatic fibrosis induced by dimethylnitrosamine administration in rats. Food Chem Toxicol 48: 3137-3143, 2010.

10. Rodrigues-Diez R, Rodrigues-Diez RR, Lavoz C, Carvajal G, Droguett A, Garcia-Redondo AB, Rodriguez I, Ortiz A, Egido J, Mezzano S and Ruiz-Ortega M: Gremlin activates the Smad pathway linked to epithelial mesenchymal transdifferentiation in cultured tubular epithelial cells. Biomed Res Int 2014: 802841, 2014.

11. Antoon JW, Nitzchke AM, Martin EC, Rhodes LV, Nam S, Wadsworth S, Salvo VA, Elliott S, Collins-Burow B, Nephew KP and Burow ME: Inhibition of p 38 mitogen-activated protein kinase alters microRNA expression and reverses epithelial-to-mesenchymal transition. Int J Oncol 42: 1139-1150, 2013.

12. Rodrigues-Díez R, Carvajal-González G, Sánchez-López E, Rodríguez-Vita J, Rodrigues Díez R, Selgas R, Ortiz A, Egido J, Mezzano S and Ruiz-Ortega M: Pharmacological modulation of epithelial mesenchymal transition caused by angiotensin II. Role of ROCK and MAPK pathways. Pharm Res 25: 2447-2461, 2008.

13. Gu L, Gao Q, Ni L, Wang M and Shen F: Fasudil inhibits epithelial-myofibroblast transdifferentiation of human renal tubular epithelial HK-2 cells induced by high glucose. Chem Pharm Bull (Tokyo) 61: 688-694, 2013.

14. Cai Q, Li BY, Gao HQ, Zhang JH, Wang JF, Yu F, Yin M and Zhang Z: Grape seed procyanidin b2 inhibits human aortic smooth muscle cell proliferation and migration induced by advanced glycation end products. Biosci Biotechnol Biochem 75: 1692-1697, 2011. 
15. O'Kane D, Jackson MV, Kissenpfennig A, Spence S, Damkat-Thomas L, Tolland JP, Smyth AE, Denton CP, Stuart Elborn J, McAuley DF and O'Kane CM: SMAD inhibition attenuates epithelial to mesenchymal transition by primary keratinocytes in vitro. Exp Dermatol 23: 497-503, 2014.

16. Islam SS, Mokhtari RB, El Hout Y, Azadi MA, Alauddin M, Yeger $\mathrm{H}$ and Farhat WA: TGF- $\beta 1$ induces EMT reprogramming of porcine bladder urothelial cells into collagen producing fibroblasts-like cells in a Smad2/Smad3-dependent manner. J Cell Commun Signal 8: 39-58, 2014.

17. Antoon JW, Nitzchke AM, Martin EC, Rhodes LV, Nam S, Wadsworth S, Salvo VA, Elliott S, Collins-Burow B, Nephew KP and Burow ME: Inhibition of p38 mitogen-activated protein kinase alters microRNA expression and reverses epithelial-to-mesenchymal transition. Int J Oncol 42: 1139-1150, 2013

18. Yeh WJ, Yang HY and Chen JR: Soy $\beta$-conglycinin retards progression of diabetic nephropathy via modulating the insulin sensitivity and angiotensin-converting enzyme activity in rats fed with high salt diet. Food Funct 5: 2898-2904, 2014.

19. Filippone EJ, Gupta A and Farber JL: Normoglycemic diabetic nephropathy: The role of insulin resistance. Case Rep Nephrol Urol 4: 137-143, 2014.

20. Kothari AN, Mi Z, Zapf M and Kuo PC: Novel clinical therapeutics targeting the epithelial to mesenchymal transition. Clin Transl Med 3: 35, 2014.

21. Srivastava SP, Koya D and Kanasaki K: MicroRNAs in kidney fibrosis and diabetic nephropathy: Roles on EMT and EndMT. Biomed Res Int 2013: 125469, 2013.

22. Fintha A, Gasparics Á, Fang L, Erdei Z, Hamar P, Mózes MM Kökény G, Rosivall $\mathrm{L}$ and Sebe A: Characterization and role of SCAI during renal fibrosis and epithelial-to-mesenchymal transition. Am J Pathol 182: 388-400, 2013.

23. Rinaldi A, Jourdes M, Teissedre PL and Moio L: A preliminary characterization of Aglianico (Vitis vinifera L. cv.) grape proanthocyanidins and evaluation of their reactivity towards salivary proteins. Food Chem 164: 142-149, 2014.
24. Agarwal C, Veluri R, Kaur M, Chou SC, Thompson JA and Agarwal R: Fractionation of high molecular weight tannins in grape seed extract and identification of procyanidin B2-3,3'-di-O-gallate as a major active constituent causing growth inhibition and apoptotic death of DU145 human prostate carcinoma cells. Carcinogenesis 28: 1478-1484, 2007.

25. Chou SC, Kaur M, Thompson JA, Agarwal R and Agarwal C: Influence of gallate esterification on the activity of procyanidin $\mathrm{B} 2$ in androgen-dependent human prostate carcinoma LNCaP cells. Pharm Res 27: 619-627, 2010.

26. Avelar MM and Gouvêa CM: Procyanidin b2 cytotoxicity to mcf-7 human breast adenocarcinoma cells. Indian J Pharm Sci 74: 351-355, 2012

27. Liu J, Zeng L, Zhao Y, Zhu B, Ren W and Wu C: Selenium suppresses lipopolysaccharide-induced fibrosis in peritoneal mesothelial cells through inhibition of epithelial-to-mesenchymal transition. Biol Trace Elem Res 161: 202-209, 2014.

28. Sakano K, Mizutani M, Murata M, Oikawa S, Hiraku Y and Kawanishi S: Procyanidin B2 has anti- and pro-oxidant effects on metal-mediated DNA damage. Free Radic Biol Med 39: 1041-1049, 2005.

29. Borthwick LA, Gardner A, De Soyza A, Mann DA and Fisher AJ: Transforming growth factor- $\beta 1$ (TGF- $\beta 1$ ) driven epithelial to mesenchymal transition (EMT) is accentuated by tumour necrosis factor $\alpha(\mathrm{TNF} \alpha)$ via crosstalk between the SMAD and NF- $\kappa$ B pathways. Cancer Microenviron 5: 45-57, 2012.

30. Tsai TH, Sun MH, Ho TC, Ma HI, Liu MY and Tsao YP: Notch prevents transforming growth factor-beta-assisted epithelial-mesenchymal transition in cultured limbal progenitor cells through the induction of Smad7. Mol Vis 20: 522-534, 2014.

31. Chen HH, Zhou XL, Shi YL and Yang J: Roles of p38 MAPK and JNK in TGF- $\beta 1$-induced human alveolar epithelial to mesenchymal transition. Arch Med Res 44: 93-98, 2013. 